\title{
Teledermatology During COVID-19: An Updated Review
}

\author{
Morgan A. Farr ${ }^{1} \cdot$ Madeleine Duvic $^{2} \cdot$ Tejas P. Joshi $^{1}$ (1) \\ Accepted: 19 March 2021 / Published online: 9 April 2021 \\ (c) The Author(s), under exclusive licence to Springer Nature Switzerland AG 2021
}

\begin{abstract}
The coronavirus disease 2019 (COVID-19) pandemic has fundamentally transformed the landscape of providing dermatologic care. In an age of lockdowns and social distancing, teledermatology (TD) has emerged as a powerful tool to deliver remote care. Here, we review literature on TD use during the pandemic to evaluate the positives and negatives of TD implementation. We especially consider the reception of TD in underserved communities and the developing world as well as the ethico-legal challenges wrought by the burgeoning utilization of this new paradigm of care. The potential of TD to occupy a more prominent role in dermatologic care in a post-COVID-19 world is also discussed.
\end{abstract}

\section{Key Points}

Teledermatology (TD) has been generally positively received during the coronavirus disease 2019 (COVID19) pandemic.

Nonetheless, numerous challenges (from access to TD dimension as well as an ethico-legal dimension) exist for TD implementation both in the US and around the world.

Solutions to circumvent these challenges have emerged during the pandemic and raise the possibility of TD occupying a more central role in the paradigm of dermatologic care.

\section{Introduction}

Teledermatology (TD) has increasingly become a popular platform for providing dermatologic care. Low cost of care, increased efficiency, and high physician and patient satisfaction are now widely recognized benefits of TD $[1,2]$.

Tejas P. Joshi

tejas.joshi@bcm.edu

1 School of Medicine, Baylor College of Medicine, 1 Baylor Plaza, Houston, TX 77030, USA

2 Department of Dermatology, MD Anderson Cancer Center, Houston, TX, USA
Additionally, TD offers greater flexibility to both the dermatologist and the patient, as care can be provided synchronously (face-to-face) in a live format and asynchronously (store-and-forward), where images obtained by the patient can be transmitted to the dermatologist [2].

The lockdown imposed by the coronavirus disease 2019 (COVID-19) pandemic has resulted in a spectacular growth of TD [3]. TD has allowed dermatologists to provide dermatological care remotely, mitigating the opportunities for COVID-19 to spread in inpatient settings [4]. In June 2020, Elsner reviewed the utilization of and attitudes towards TD during the COVID-19 days. Overall, the results of the review indicated an increased reliance on TD during the pandemic and overall positive attitudes toward this new model of care [5]. Here, we present an updated review of TD during the pandemic.

\section{Discussion}

\subsection{Promises and Positives}

In addition to minimizing the spread of COVID-19, the implementation of TD during the COVID-19 pandemic has exposed unique benefits to both patients and dermatologists. In the US, an historical challenge dermatology has faced is access to care. Prior to COVID-19, a study of the nationwide distribution of dermatologists showed that metropolitan areas have 4.03 dermatologists per 100,000 residents, whereas rural areas have only 3.06 dermatologists per 100,000 residents [6]. This can be compared with a similar 
study that showed an average of just over 50 primary care physicians per 100,000 residents in both rural and urban areas [7]. The comparison of these studies makes it hard to ignore a serious gap in access to in-person care that dermatology has faced, both before and during COVID-19. Although the social distancing restrictions put into place due to COVID-19 have further imposed a barrier to in-person dermatologic care, dermatologists have increasingly used TD in order to expand care. In fact, the US lifted restrictions on TD, allowing dermatologists to practice across state lines during the COVID-19 pandemic [8]. In addition, the extended use of TD promises a greater access to care for patients who experience socioeconomic barriers such as lack of childcare, social anxiety, inaccessible transportation, and difficult work schedules [9], due to the fact that patients are able to access TD visits from the comfort of their home.

Aside from improved access to care, TD during the COVID-19 pandemic has shown an advantage of being both time- and cost-effective for patients and physicians. As previously mentioned, patients living in rural areas have limited access to dermatologists [6], often being forced to travel great distances for in-person dermatology appointments. By providing at-home care, TD cuts travel costs for patients and eliminates time spent driving to a doctor's office [10]. In addition to reduced travel costs, a 2019 study in Philadelphia revealed that TD reduces medical costs for patients because timely access to care provided by TD reduces urgent care and emergency department visits. This same study showed that the reimbursement for TD visits is lower for physicians, as TD cuts visit costs by US $\$ 10-\$ 80$ compared with in-person visits [11]. When this study was conducted prior to COVID-19, insurance agencies provided lower reimbursement for TD visits over in-person visits [12]. To urge physicians to practice TD during COVID-19, insurance companies have begun to give physicians payment parity for TD visits, maintaining physician salary [9]. This payment parity has reversed the side effect of decreased physician compensation for TD services. In addition to saving money, asynchronous TD visits are generally shorter than inperson appointments, saving waiting and visit times for the patient and allowing physicians to schedule more appointments [13]. Although shorter visits are thought to sacrifice quality of care, TD appointments are thorough due to the fact that store-and-forward TD allows physicians to review patient photos and history before their visit [2]. However, it should be acknowledged that while shorter consultations are a benefit of asynchronous TD, time saving (on the part of the dermatologist) has not been reported for synchronous TD.

Overall, the previously mentioned advantages of TD in the COVID-19 era have led to high patient satisfaction with dermatologic care. A number of studies from Italy underscore the promises of TD. In one Italian study that surveyed patients receiving TD treatment for acne during COVID-19,
$92.3 \%$ of the 52 patients surveyed were satisfied with the attention given to them by their dermatologist, and $86.5 \%$ of patients were satisfied with the length of the visit [14]. Similarly, high satisfaction with acne care provided via TD was reported in another Italian study of 150 TD consults [15]. In yet another Italian study of 300 subjects, increased adherence to treatment in patients receiving reminder text messages (compared with patients not receiving any reminder messages) was reported and suggests the potential for a TD intervention to improve the continuity of care [16]. Furthermore, in a 2020 study conducted in the UK among 60 patients with skin cancer, $80 \%$ of patients said they would recommend TD to a friend or family member. Questions to determine satisfaction included cost, attention to care, methods of taking photos of lesions, and time effectiveness. This same study also interestingly revealed that younger patients were more likely to report willingness to use TD in the future, and patients who were generally healthy were more satisfied with TD than those in poor health [17]. The latter implications of this study reveal the importance of TD not replacing in-person visits altogether, as TD has drawbacks that will be discussed next.

\subsection{Challenges and Solutions}

The challenges of TD, for patients as well as physicians, have been well-described in the literature [18] and the COVID-19 pandemic has brought many of these challenges to the spotlight. From the patient standpoint, while TD has afforded patients the ability to receive diagnoses without risking a clinic visit, the pandemic has limited access to clinical procedures. This has placed patients in a quagmire: all too aware of their diagnoses, patients must anxiously wait to seek procedural care [19].

The dermatologist too has not been spared from the challenges brought along by a transition to TD. Notably, the study by Bhargava et al., evaluating reasons for mental distress among dermatologists during the pandemic found TD utilization to be the most significant predictor of mental distress. Perhaps this stress has been precipitated by the lack of a technical infrastructure to support a fully functioning TD service and/or uncertainty regarding reimbursements for online appointments [20]. Moreover, while TD affords the opportunity to inspect and diagnose lesions pertinent to the chief complaint, other concerning lesions that would have been otherwise caught during a complete skin examination may be missed. Deacon and Madigan report a case that underscores this very issue: while a TD visit was accurately able to diagnose a herpes infection, another serious lesion was narrowly missed. As this particular patient was located in a low-risk site and did not exhibit any COVID-19 symptoms, the dermatology team decided to follow-up with an in-person consult, during which they discovered another 
lesion (unrelated to the patient's primary complaint) on the patient's left lower leg that was biopsied and identified as malignant melanoma [21]. Of course, had this patient been in a high-risk setting or have reported characteristic COVID19 symptoms, perhaps the in-person consult may have never taken place and the diagnosis of melanoma altogether missed. It is certainly troubling to imagine how many such diagnoses may have been missed as the world transitioned to TD during COVID-19.

Moreover, making diagnoses virtually has been difficult. In the store-and-forward model of dermatologic care, patients may submit subpar photographs that render making a diagnosis almost impossible. In Madrid, a pilot study to analyze the viability of TD during the pandemic found that only $52.1 \%$ of all submitted images were of sufficient quality, and in approximately $10 \%$ of cases, a diagnosis could not be rendered either because the image quality was poor or because the submitted image did not pertain to the chief complaint [22]. In another study to evaluate patient attitudes towards the technical experience of synchronous TD during COVID-19, Pearlman et al. encountered the same problem, noting that about one in three patients reported that their dermatologist was only able to see their skin to a 'poor' or 'acceptable' degree. Significantly, while their study found overwhelming satisfaction with TD, $68.7 \%$ of patients indicated a preference for in-person consultation, as opposed to $\mathrm{TD}$, for their next appointment [23].

It is also worth noting that dermatology residents and medical students have seen their training hampered by the pandemic as TD visits replaced in-person encounters [24]. Additionally, a UK-wide survey administered in August 2020 uncovered experience with TD to be a serious lacuna for dermatology trainees, with 58\% of residents reporting feeling only 'slightly confident' providing care via TD [25].

Nevertheless, these challenges are not without solutions. Choi et al. outlined solutions to many of the challenges presented above: to ameliorate patient anxiety, a triaging system to identify and treat patients with the most serious lesions; to ease the physician strain with tenuous technical infrastructure and nebulous reimbursement protocols, government policies to strengthen TD platforms and implementation of clear mechanisms to ensure appropriate physician compensation; to combat poor visual resolution, use of a gray card as reference during photo taking (corrects for differences in lighting) and a medley of other camera hacks [26].

Furthermore, during the very midst of the pandemic, innovative new technologies have been introduced to tackle the limitations of TD. In particular, artificial intelligence (AI) technologies promise to yield seminal solutions. In Boston, Su et al. [27] developed a feedback algorithm that outputs 'smart phrases' that prompt the patient to resubmit new images if the original images were of inadequate quality. This algorithm promises to increase the efficiency of TD by eliminating images that would not have yielded a diagnosis. Moreover, AI has been hinted as a tool to stratify patients based on risk of lesion in order to treat those with the most pressing disease first; of course, this would mitigate the anxiety of patients who have more serious lesions [28]. Apart from $\mathrm{AI}$, other creative strategies have been suggested to circumvent the limitations of TD. In October 2020, Blum and Menzies [29] described a technique for home dermoscopy that involved nothing more than disinfectant spray, cooking oil, and water, and in January 2021, Kaliyadan et al. [30] reported a technique only requiring hand sanitizer. Indeed, while both reports concede that clinical dermoscopy is superior to these amateur methods, these strategies nonetheless offer creative solutions to situations where TD may be the only venue to deliver dermatologic care.

Finally, when it comes to TD and medical education, medical trainees can still see patients virtually (under the supervision of their attendings) [31] and appreciate the transition to TD not as an obstacle but rather as an opportunityan opportunity to gain proficiency in a model of care that is likely to only gain traction in the coming years.

Despite these solutions to circumvent the barriers of TD implementation, it is important to realize that the inability to provide procedural care in a strictly TD model of care represents a limitation of TD that is not amenable to any solutions. Indeed, the lack of access to procedural care following TD consultation was recognized early on during the pandemic [3] and remains a major limitation of the TD platform.

\subsection{Perspectives of Underserved Communities and Developing Nations}

There is a paucity of literature considering the attitudes of underserved communities towards the transition to TD during COVID-19. We were able to identify three studies that considered racial/ethnic variations in TD utilization, but the results of these studies were inconsistent. A Boston study found that Spanish-speaking patients scheduled fewer TD appointments during the pandemic compared with total dermatology visits scheduled prior to the COVID-19 days. Also significant, as opposed to their non-Spanish speaking counterparts, Spanish-speaking patients were less likely to have linked their medical record to an email or have created an online account preceding their TD visit [32]. However, another Massachusetts study (conducted between May 2019 and June 2020) found no significant racial/ethnic differences in TD utilization, and demonstrated that the no-show rate for TD visits was markedly lower than that for clinic visits and that the most precipitous reduction in no-show rates was actually among the Black/African American, LatinX, and non-English speaking patients [33]. Of course, as part of the data for this study were collected prior to the pandemic, it is 
possible that disparities in TD access during the COVID-19 months may have been diminished by data from the preCOVID-19 days. Another Boston study (limited to data only from the COVID-19 months) surveying patient comfort with TD found no racial differences in preference for TD use, but noted Hispanic ethnicity to be significantly associated with hesitancy in utilizing TD, as non-Hispanic patients demonstrated an approximately 12 -fold higher likelihood of preferring a TD visit to an in-person visit [34].

Thus, current literature on the perspectives of underserved communities towards the TD transition is ambiguous. The current studies are also narrow in geographic scope as they were all conducted in Massachusetts. Additionally, no studies have specifically investigated the potential inequities in access to TD during COVID-19 among rural Americans (although hesitancy to utilize telehealth in general has been noted [35]). There is certainly a need for more studies on the topic, not just so that potential inequities can be identified but also so that strategies can be developed to expand TD access.

In the developing world, the attitudes towards TD transition have been varied, and, once again, the literature evaluating the transition to TD is scant. In the Middle East, we found literature from Turkey, Egypt, and Iran that considered the perspectives towards TD. A study in Turkey reported positive perspectives towards TD, with $86 \%$ of dermatologists in private practice reporting positive responses from their patients [36]. Another study conducted at a teaching hospital in Turkey found the TD model to be suitable for $72.8 \%$ of the study population, but the authors conceded that TD access is not yet universal and the legal framework for providing this new mode of care is still nascent [37]. In Egypt, Mostafa and Hegazy reported high patient satisfaction with TD and recognized the utility of TD in triaging patients in both private practice and public hospitals. Overall, they concluded TD to be a viable platform to deliver care in Egyptian dermatology practice [38]. In Iran, in the absence of an established TD platform, WhatsApp emerged as a powerful tool to reach patients receiving care at a teaching hospital. Additionally, dermatology residents reached out to high-risk patients via phone call and these check-ins were deeply appreciated by patients. Nevertheless, the absence of an established technical and legal infrastructure to support TD has undermined the utility of TD in Iran [39]. From Saudi Arabia, Kaliyadan et al. [40] recognized a very important cultural limitation to TD: patients, especially female, may not be comfortable with TD consultations for cultural/religious reasons. It is possible that a similar concern exists in the rest of the Middle East and illustrates the importance of considering how cultural milieu can impact TD utilization.

In India, the general sentiment towards TD seems to be that of tepid acceptance. For a country with a serious paucity of dermatologists, the rise of TD during the COVID-19 crisis has been viewed as a promising opportunity to revolutionize dermatologic care [41]. Nonetheless, access to internet connection remains a hindrance of the TD platform. In a study of 84 patients evaluated by TD during COVID-19, Doddaballapur et al. [42] acknowledged access to stable internet connection to be a constraining factor for TD utilization. Additionally, technology illiteracy persists among the Indian populace and constitutes another major hurdle to the TD implementation in India [41]. Indeed, a study considering the profile of TD consultations during the pandemic reported that $84 \%$ of TD users resided in urban areas, suggesting that roadblocks still persist in India towards more widespread implementation of TD [43].

In Nepal, TD was successfully deployed during the pandemic to identify a case of cutaneous leishmaniasis [44] and a case of carbamazepine-induced toxic epidermal necrolysis [45]. Notably, both these cases were from rural Nepal and indicate the potential for TD to penetrate beyond urban centers.

In Latina America, we found two studies that dealt with the impact of TD during the pandemic. In Argentina, the results of a study surveying patient and physician attitudes towards TD during the COVID-19 crisis are particularly noteworthy. All patients who responded to the satisfaction survey responded positively and all dermatologists surveyed were pleased with TD. It is worth noting that $47.5 \%$ of patients in the study did not have health insurance, suggesting that, in the case of Argentina, further implementation of TD may expand the access to dermatologic care [46]. In Brazil, Bimbi et al. [47] reported the utility of TD during the pandemic in solving two cases of crusted scabies, but their case report represents more a proof of concept rather than a national survey.

Despite the aforementioned studies, a complete picture of the global attitudes towards TD during the pandemic is glaringly incomplete. While Oaku and Anaba [48] report the transition to TD in Sub-Saharan Africa to have been plagued by poor internet connection, lack of ubiquitous smart phone access, and an aging populace, there is otherwise no literature considering the African experience with TD during COVID-19. Also notable, we did not find any studies from East or Southeast Asia considering their experience with the TD transition. Additionally, some of the experience with TD in the developing world has been captured by anecdotal case reports, and there is an overall scarcity of large-scale studies that analyze the satisfaction with TD. An attempt to gauge the global satisfaction with TD was made in December 2020, but the study was largely Eurocentric, with dermatologists from Asia, South America, and Africa comprising $<15 \%$ of the study population [49]. 


\subsection{Ethico-Legal Challenges}

The increased use of TD during the COVID-19 pandemic has imposed ethical and legal challenges on physicians. Historically, dermatologists in the US have faced challenges regarding reimbursement, as TD reimbursement policies traditionally vary from state to state [50]. Overall, Medicare reimbursement is typically US $\$ 15$ for virtual patient check-ins, compared with US\$22-\$148 for in-person visits, depending on the level of care [13]. The reduced reimbursement for TD visits has generally driven clinicians away from practicing virtual care before the pandemic. In fact, prior to COVID-19, only 16 states enacted payment parity laws, which reimburse physicians for TD visits as regular office visits [12]. Since in-person visits carry the risk of spreading COVID-19, Medicare, state Medicaid programs, and private payers have temporarily issued waivers to provide payment parity between TD visits and in-person visits in order to slow the spread of the virus [8]. Expanded payment parity regulations may encourage TD in the midst of the COVID-19 era, but there is no clear timeframe for when the payment parity waivers will be retracted, leading to an increased likelihood that dermatologists will continue to face the challenge of reimbursement post-COVID-19.

In addition to the barrier of reimbursement, physicians were legally limited to providing TD services to patients residing in the state in which they were licensed prior to COVID-19 [51]. This restriction prevented dermatologists from practicing telemedicine across state lines, therefore reducing the number of patients reached by TD. This predicament was recognized, as the possibility of federal or telehealth-specific licensure had been discussed prior to the pandemic. Unfortunately, policymakers opposed this idea, further limiting patient access to dermatologic care [51]. Because COVID-19 necessitated quarantining and exponentially increased the demand for TD, the majority of states (with the exception of Alabama, Delaware, Iowa, Michigan, and Ohio) lifted these restrictions, allowing dermatologists to treat and bill patients in any location [52]. The relaxation of restrictions has temporarily increased the patient pool for TD, but dermatologists still face the uncertainty of when state licensure guidelines will be re-enacted.

Unlike the aforementioned challenges faced by TD, the legal barrier of malpractice associated with telemedicine has not been resolved with the COVID-19 pandemic. With the increasing popularity of practicing telemedicine across state lines during COVID-19 [13], it is important to note that not all malpractice insurance covers telemedicine, and some malpractice insurance does not cover practice across state lines [53]. A common lack in malpractice insurance forces dermatologists to confront the medical liability risks of TD. One of these risks is a common challenge that may lead to misdiagnosis - the quality of photos taken by patients. Store-and-forward TD relies on the transmission and storage of photos, and a review written during COVID19 showed that smartphones are a widespread method of taking clinical photos [54]. Another 2020 study revealed that smartphones with automatic camera settings produce oversaturated photos, making it difficult to determine possible malignancy based on color of lesions [55]. In addition to the implied risk of misdiagnosis, taking and storing photos on personal smartphones is not considered a secure way of storing patient information and can lead to Health Insurance Portability and Accountability Act (HIPAA) violations [54].

HIPAA violations have always posed challenges for TD, as barriers to telecare include obtaining consent for visits, keeping patient information private, and finding secure communication platforms. The lack of secure communication platforms available for telehealth has been addressed by the Office for Civil Rights since the beginning of the pandemic, as it is recognized as one of the main barriers to providing secure care. The Office for Civil Rights has allowed dermatologists to use alternative methods for video visits such as FaceTime, Facebook Messenger, WhatsApp, Skype, and Google Hangouts since the beginning of the pandemic [8]. These applications, specifically Facebook Messenger, require users to agree to their privacy statement and do not offer encrypted messaging by default [56], leaving patient information vulnerable to data breaches. Lack of security of patient information and an increased risk for HIPAA violations are core barriers that prevent the practice of telehealth. To slow the spread of COVID-19 and encourage telehealth visits, the Office of Civil Rights decided to waive HIPAA violations that occur while attempting to provide TD services in good faith during the COVID-19 pandemic [9]. However, with no clear idea of when this policy will end and an insufficient variety of secure communication platforms, HIPAA violations will likely be a barrier to TD in the future.

\subsection{Looking Forward: Teledermatology in a Post-COVID-19 World}

Despite hopes of an end to the COVID-19 pandemic, TD remains an innovative tool in the future care of patients. In order to maintain popularity of TD visits in a postCOVID-19 world, standardized telemedicine training must be implemented for both physician confidence and patient safety. As of right now, less than half of dermatology residency programs in the US have a curriculum that incorporates TD [57]. Even more concerning, there is not a standardized curriculum in place for teaching telemedicine across the US [58]. The lack of standardized telemedicine training has become evident during the rapid transition to TD during the COVID-19 pandemic, as many providers continue to voice telemedicine concerns, such as risk of compromised patient care [59]. These concerns could be minimized with 
a standardized and required curriculum for TD implemented in residency, allowing physicians to increase confidence in patient care and be more willing to practice telemedicine. For example, a pre-COVID-19 study at the University of Iowa implemented a three-module telemedicine educational course for second-year medical students. The students who participated in the module reported improved telemedicine knowledge and confidence with patients [60]. Since COVID19 , multiple reviews have also suggested goals for TD training. Common goals include education on access to care, cost effectiveness, patient experience, and physician experience [59]. Without education in these areas, the future of TD may be challenged by a need for training and improved patient care.

Another roadblock TD may face in a post-COVID-19 world is a need for healthcare equity to underserved populations. Although relaxed state practicing laws have improved access to TD for many [8], not all patients are able to benefit from these changes. For example, a study published in 2020 of Medicare beneficiaries discovered that $41.4 \%$ of Medicare patients lack access to a computer with high-speed internet at home, and $40.9 \%$ lack a smartphone with a wireless internet plan; $26.3 \%$ of patients lack either form of telemedicine access. The proportion of Medicare beneficiaries lacking access to care was found to be higher in communities of color, patients over 85 years of age, and those with low socioeconomic status [61]. The results of this study indicate that inequalities in healthcare still exist, and until these inequalities are resolved, TD will not be able to adequately provide care for all. In another 2020 study of cancer patients in rural Virginia, only 58\% had broadband access necessary for a telemedicine visit, and it took an average of $29.6 \mathrm{~min}$ to drive to the nearest telemedicine facility. This study proposed that the ideal solution for equity would be to expand broadband internet access across rural America, but this suggestion is unattainable due to cost. A more plausible solution proposed by this study is to use local libraries for telemedicine visits. Libraries are commonly distributed across the US, therefore finding secure areas within local libraries for TD visits would help increase access to care within rural and lowincome areas [62].

Regardless of the hindrances to telemedicine in the future, technological advances in TD provide a positive outlook on dermatology in a post-COVID-19 world. Recent advancement of AI smartphone applications (apps) allow patients to take photos of skin lesions and receive a speculative diagnosis [63]. A 2020 review of dermatologic AI smartphone apps stated that AI allows skin cancer patients to be treated more effectively and quickly, which is significant given the increasing incidence of skin cancer. The same review also emphasized the importance of a dermatologist reviewing the classifications of lesions as malignant or benign, since AI has been associated with inaccuracy [64]. This conclusion is emphasized by a study completed during COVID-19, where an AI screening tool was used to aid dermatologists in their diagnosis. In this study, AI was shown to be useful in only $11.8 \%$ of cases [63]. The significance of these studies combined is that the future of $\mathrm{AI}$ lies in aiding clinicians to improve patient care, not in replacing the job of dermatologists altogether. With the help of AI, dermatologists can therefore make quicker diagnoses and see more patients virtually. However, in order for smartphone apps to successfully assist dermatologists, patients must be willing to use AI services. In a recent study of MoleMe, an AI cutaneous pigment evaluator developed in Taiwan, 90\% of participants showed satisfaction with the app. This satisfaction was consistent across varying genders and age groups [65]. If combined with success in improving technologic healthcare equity, smartphone apps incorporating dermatologic AI could be groundbreaking in the diagnosis and care of patients via TD.

\section{Conclusion}

The rise of TD during the COVID-19 pandemic has illuminated a path for improved dermatologic care. TD has shown many benefits, such as time- and cost-effectiveness and high patient satisfaction. Despite these benefits, many detractors to TD have been exposed through the increased use of telemedicine in the COVID-19 era. Dermatologists have been faced with increased stressors, including lack of technological support and standardized training, as well as risk for incorrect or incomplete diagnoses due to lowquality images submitted by patients in store-and-forward dermatology. Solutions have been suggested (and implemented) for many of these challenges. Many of the ethicolegal challenges faced by TD before COVID-19 have also been resolved during the pandemic, such as payment parity for telehealth visits, legalities of practicing across state lines, and risk of HIPAA violations for using unsecure communication platforms. Unfortunately, these resolutions are predicted to be only temporary, posing a threat to the increased use of TD in the post-COVID-19 era. Studies on global attitudes of underserved communities and developing nations reveal a general positive outlook on TD during COVID-19, but are overarchingly incomplete and deficient in perspectives from Africa and East/Southeast Asia. Overall, TD has enabled improved patient care, but future research and improvements on standardized telehealth training, internet access, and AI will allow TD to continue to gain traction after the resolution of the COVID-19 pandemic. We summarize our findings in Table 1. 
Table 1 Perspectives toward teledermatology during the COVID-19 pandemic

Promises of TD

Detractors of TD

Barriers to TD implementation in the developing world

Ethico-legal challenges of TD
Increase in access to care in rural areas

Time and cost saving for patients

Time saving for physicians (with asynchronous TD)

Potential to establish continuity of care

Inability to perform procedures

Inability to perform full body examinations

Poor video/image quality

Inexperience of medical students and residents with the TD platform

Potential disparities in TD utilization among underserved communities

Tenuous internet access

Technological illiteracy

Lack of a legal framework to sustain long-term TD practice

Cultural barriers

Payment parity between TD consultations and in-person visits

Restrictions to practicing across state lines

Encryption of the TD platform

Malpractice insurance coverage of TD visits

COVID-19 coronavirus disease 2019, TD teledermatology

\section{Declarations}

Funding Not applicable.

Conflicts of interest Morgan A. Farr, Madeleine Duvic, and Tejas P. Joshi declare no conflicts of interest.

Availability of data and material Articles used in this review are available in the public domain.

Code availability Not applicable.

Ethics approval Not applicable.

Consent to participate Not applicable.

Consent to publish Not applicable.

Author contributions TPJ conceived the idea for this review; TPJ and MAF performed the literature search and drafted the manuscript; and MD critically revised the work. All authors read and approved the submitted version.

\section{References}

1. Eedy DJ, Wootton R. Teledermatology: a review. Br J Dermatol 1951. 2001;144(4):696-707. https://doi.org/10.1046/j.1365-2133. 2001.04124.x.

2. Lee JJ, Lee JJ, English JC III, English JC III. Teledermatology: a review and update. Am J Clin Dermatol. 2018;19(2):253-60. https://doi.org/10.1007/s40257-017-0317-6.

3. Perkins S, Cohen JM, Nelson CA, Bunick CG. Teledermatology in the era of COVID-19: experience of an academic department of dermatology. J Am Acad Dermatol. 2020;83(1):e43-4. https://doi.org/10.1016/j.jaad.2020.04.048.

4. Gupta R, Ibraheim MK, Doan HQ. Teledermatology in the wake of COVID-19: advantages and challenges to continued care in a time of disarray. J Am Acad Dermatol. 2020;83(1):168-9. https://doi.org/10.1016/j.jaad.2020.04.080.

5. Elsner P. Teledermatology in the times of COVID-19-a systematic review. J Dtsch Dermatol Ges. 2020;18(8):841-5. https://doi.org/10.1111/ddg.14180.

6. Feng H, Berk-Krauss J, Feng PW, Stein JA. Comparison of dermatologist density between urban and rural counties in the United States. JAMA Dermatol. 2018;154(11):1265-71. https:// doi.org/10.1001/jamadermatol.2018.3022.

7. Machado SR, Jayawardana S, Mossialos E, Vaduganathan M. Physician density by specialty type in urban and rural counties in the US, 2010 to 2017. JAMA Netw Open. 2021;4(1):e2033994. https://doi.org/10.1001/jamanetworkopen. 2020.33994.

8. Dermatologists can use telemedicine during COVID-19 outbreak. 2021. https://www.aad.org/member/practice/telederm/toolkit. Accessed 30 Jan 2021.

9. Pulsipher KJ, Presley CL, Rundle CW, Rietcheck HR, Millitelo M, Dellavalle RP. Teledermatology application use in the COVID-19 era. Dermatol Online J. 2020;26:12. https://escholarship.org/uc/ item/1fs0m0tp. Accessed 30 Jan 2021.

10. Beer J, Hadeler E, Calume A, Gitlow H, Nouri K. Teledermatology: current indications and considerations for future use. Arch Dermatol Res. 2020. https://doi.org/10.1007/s00403-020-02145-3 ((Epub 19 Oct 2020)).

11. Yang X, Barbieri JS, Kovarik CL. Cost analysis of a store-andforward teledermatology consult system in Philadelphia. J Am Acad Dermatol. 2019;81(3):758-64. https://doi.org/10.1016/j. jaad.2018.09.036.

12. Bressler MY, Siegel DM, Markowitz O. Virtual dermatology: a COVID-19 update. Cutis. 2020;105(4):163-4.

13. Puri P, Yiannias JA, Mangold AR, Swanson DL, Pittelkow MR. The policy dimensions, regulatory landscape, and market 
characteristics of teledermatology in the United States. JAAD Int. 2020;1(2):202-7. https://doi.org/10.1016/j.jdin.2020.09.004.

14. Ruggiero A, Megna M, Annunziata MC, et al. Teledermatology for acne during COVID-19: high patients' satisfaction in spite of the emergency. J Eur Acad Dermatol Venereol. 2020;34(11):e662-3. https://doi.org/10.1111/jdv.16746.

15. Villani A, Megna M, Scalvenzi M, Fabbrocini G, Ruggiero A. Teledermatology and chronic skin diseases: real life experience in a Southern Italian Dermatologic Centre. Dermatol Ther. 2020;33(6):e13839. https://doi.org/10.1111/dth.13839.

16. Marasca C, Ruggiero A, Fontanella G, Ferrillo M, Fabbrocini $\mathrm{G}$, Villani A. Telemedicine and support groups could be used to improve adherence to treatment and health-related quality of life in patients affected by inflammatory skin conditions during the COVID-19 pandemic. Clin Exp Dermatol. 2020;45(6):749-749. https://doi.org/10.1111/ced.14245.

17. Nicholson P, Macedo C, Fuller C, Thomas L. Patient satisfaction with a new skin cancer teledermatology service. Clin Exp Dermatol. 2020;45(6):691-8. https://doi.org/10.1111/ced.14191.

18. Whited JD. Teledermatology research review. Int J Dermatol. 2006;45(3):220-9. https://doi.org/10.1111/j.1365-4632.2004. 02427.x.

19. Berman HS, Shi VY, Hsiao JL. Challenges of teledermatology: lessons learned during COVID-19 pandemic. Dermatol Online J. 2020;26:11.

20. Bhargava S, Sarkar R, Kroumpouzos G. Mental distress in dermatologists during COVID-19 pandemic: assessment and risk factors in a global, cross-sectional study. Dermatol Ther. 2020;33(6):e14161. https://doi.org/10.1111/dth.14161.

21. Deacon DC, Madigan LM. Inpatient teledermatology in the era of COVID-19 and the importance of the complete skin examination. JAAD Case Rep. 2020;6(10):977-8. https://doi.org/10.1016/j.jdcr. 2020.07.050.

22. Sendagorta E, Servera G, Nuño A, Gil R, Pérez-España L, Herranz P. Direct-to-patient teledermatology during COVID-19 lockdown in a health district in Madrid, Spain: the EVIDE-19 pilot study. Actas Dermosifiliogr. 2020. https://doi.org/10.1016/j.ad. 2020.11.020 ((Epub 11 Dec 2020)).

23. Pearlman RL, Le PB, Brodell RT, Nahar VK. Evaluation of patient attitudes towards the technical experience of synchronous teledermatology in the era of COVID-19. Arch Dermatol Res. 2021. https://doi.org/10.1007/s00403-020-02170-2 ((Epub 5 Jan 2021)).

24. Viviani F, Ferrari T, Mussi M, Zengarini C, Orioni G. Dermatology residents and COVID-19: life behind the frontlines. Clin Exp Dermatol. 2021;46(1):169-70. https://doi.org/10.1111/ced.14365.

25. Lowe A, Pararajasingam A, Goodwin RG. A paradigm shift in trainee confidence in teledermatology and virtual working during the COVID-19 pandemic: results of a follow-up UK-wide survey. Clin Exp Dermatol. 2021;46(3):544-7. https://doi.org/10.1111/ ced.14498.

26. Choi E, Mak WK, Law JY, Santos D, Quek SC. Optimizing teledermatology: looking beyond the COVID-19 pandemic. Int $\mathbf{J}$ Dermatol. 2021;60(1):119-21. https://doi.org/10.1111/ijd.15272.

27. Su MY, Trefrey BL, Smith GP, Das S. Online portal-based system for improving patient-generated photographs for teledermatology. Dermatol Ther. 2020;33(6):e14453. https://doi.org/10.1111/dth. 14453.

28. Puri P, Comfere N, Pittelkow MR, Bezalel SA, Murphree DH. COVID-19: An opportunity to build dermatology's digital future. Dermatol Ther. 2020;33(6):e14149. https://doi.org/10.1111/dth. 14149.

29. Blum A, Menzies M. Home dermoscopy during the COVID-19 pandemic. Dermatol Pract Concept. 2020;10(4):e2020091. https:// doi.org/10.5826/dpc.1004a91.
30. Kaliyadan F, Jayasree P, Ashique KT. Drop dermoscopy for teledermatology. J Am Acad Dermatol. 2021;84(1):e25-6. https:// doi.org/10.1016/j.jaad.2020.07.119.

31. Mufti A, Maliyar K, Sachdeva M, Doiron P. Modifications to dermatology residency education during the COVID-19 pandemic. J Am Acad Dermatol. 2020;83(3):e235-6. https://doi.org/10.1016/j. jaad.2020.05.106.

32. Blundell AR, Kroshinsky D, Hawryluk EB, Das S. Disparities in telemedicine access for Spanish-speaking patients during the COVID-19 crisis. Pediatr Dermatol. 2020. https://doi.org/10. 1111/pde.14489 ((Epub 27 Dec 2020)).

33. Franciosi EB, Tan AJ, Kassamali B, O'Connor DM, Rashighi M, LaChance AH. Understanding the impact of teledermatology on no-show rates and health care accessibility: a retrospective chart review. J Am Acad Dermatol. 2021;84(3):769-71. https://doi.org/ 10.1016/j.jaad.2020.09.019.

34. Pannu S, Nguyen BM, Yang F-SC, Rosmarin D. Predictors of patient experience with teledermatology in setting of COVID-19 pandemic in a single medical center. Int J Dermatol. 2020. https:// doi.org/10.1111/ijd.15394 ((Epub 31 Dec 2020)).

35. Jaffe DH, Lee L, Huynh S, Haskell TP. Health inequalities in the use of telehealth in the United States in the Lens of COVID-19. Popul Health Manag. 2020;23(5):368-77. https://doi.org/10.1089/ pop.2020.0186.

36. Esme P, Akoglu G, Erbil H. Medical and socioeconomic challenges of private dermatocosmetology clinics during COVID19 pandemic: a survey from Turkey. J Cosmet Dermatol. 2020;19(12):3160-5. https://doi.org/10.1111/jocd.13801.

37. Temiz SA, Dursun R, Daye M, Ataseven A. Evaluation of dermatology consultations in the era of COVID19. Dermatol Ther. 2020;33(5):e13642. https://doi.org/10.1111/dth.13642.

38. Mostafa PIN, Hegazy AA. Dermatological consultations in the COVID-19 era: is teledermatology the key to social distancing? An Egyptian experience. J Dermatol Treat. 2020. https://doi.org/ 10.1080/09546634.2020.1789046 ((Epub 7 Jul 2020)).

39. Daneshpazhooh M, Mahmoudi H. COVID-19: the experience from Iran. Clin Dermatol. 2020. https://doi.org/10.1016/j.clind ermatol.2020.12.009 ((Epub 15 Dec 2020)).

40. Kaliyadan F, Al Ameer A, Al AQ. Telemedicine practice in saudi arabia during the COVID-19 pandemic. Cureus. 2020;12(12):e12004.

41. Ashique K, Kaliyadan F. Teledermatology in the wake of COVID19 scenario: an Indian perspective. Indian Dermatol Online J. 2020;11(3):301-6. https://doi.org/10.4103/idoj.IDOJ_260_20.

42. Doddaballapur S, Bangera A, Kaujalgi R, Dongare A. Teledermatology during COVID era: our experience. Clin Dermatol Rev. 2021;5(1):20-3. https://doi.org/10.4103/CDR.CDR_83_20.

43. Gupta M, Bhargava S. The profile of teledermatology consultations during the COVID-19 pandemic: an observational study. Dermatol Online. 2020;11(Suppl 2):10-2.

44. Paudel V. Tele-dermatology in clinical management of suspected cutaneous leishmaniasis in COVID-19 pandemic. Nepal J Dermatol Venereol Leprol. 2020;18(1):91-2.

45. Paudel V, Chudal D. Carbamazepine-induced toxic epidermal necrolysis managed by mobile teledermatology in COVID-19 pandemic in rural Nepal. Case Rep Dermatol Med. 2020. https:// doi.org/10.1155/2020/8845759.

46. Debernardi ME, Bordón MP, Campastri A, et al. TeLEdermatología en pandemia de COVID-19 en un hospital público. Med B Aires. 2020;80:80.

47. Bimbi C, Wollina U, Kyriakou G, Dalla Lana DF, Ramos M. Basic teledermatology solving two cases of crusted scabies. Dermatol Ther. 2020;33(6):e14214. https://doi.org/10.1111/dth.14214.

48. Oaku I, Anaba EL. The impact of COVID-19 on the practice of dermatology in sub-Saharan Africa. Dermatol Ther. 
2020;2020:e14642. https://doi.org/10.1111/dth.14642 ((Epub 5 Dec 2020)).

49. Moscarella E, Pasquali P, Cinotti E, Tognetti L, Argenziano G, Rubegni P. A survey on teledermatology use and doctors' perception in times of COVID-19. J Eur Acad Dermatol Venereol. 2020;34(12):772-e773. https://doi.org/10.1111/jdv.16843.

50. Campagna $\mathrm{M}$. Teledermatology: an updated overview of clinical applications and reimbursement policies. J Am Acad Dermatol. 2018;79(3 suppl 1):AB280. https://doi.org/10.1016/j.jaad.2018. 05.1109 .

51. Young JD, Borgetti SA, Clapham PJ. Telehealth: exploring the ethical issues. DePaul J Health Care Law. 2018;16(3):2.

52. US States and Territories Modifying Requirements for Telehealth in Response to COVID-19. Federation of State Medical Boards. 2021. https://www.fsmb.org/siteassets/advocacy/pdf/states-waivi ng-licensure-requirements-for-telehealth-in-response-to-covid-19. pdf. Accessed 1 Feb 2021.

53. Fields BG. Regulatory, legal, and ethical considerations of telemedicine. Sleep Med Clin. 2020;15(3):409-16. https://doi.org/ 10.1016/j.jsmc.2020.06.004

54. Manuelyan K, Shahid M, Vassilev V, Drenovska K, Vassileva S. Direct patient-to-physician teledermatology: not a flash in the pan(demic). Clin Dermatol. 2020. https://doi.org/10.1016/j.clind ermatol.2020.12.011(Epub24Dec2020).

55. Dugonik B, Dugonik A, Marovt M, Golob M. Image quality assessment of digital image capturing devices for melanoma detection. Appl Sci. 2020;10(8):2876. https://doi.org/10.3390/ app10082876.

56. Is Facebook Messenger HIPAA Compliant? HIPAA Journal. 2017. https://www.hipaajournal.com/facebook-messenger-hipaacompliant/. Accessed 30 Jan 2021.

57. Song E, Amerson E, Twigg A. Teledermatology in medical and continuing education. Curr Dermatol Rep. 2020;9:136-40. https:// doi.org/10.1007/s13671-020-00304-3.
58. Chike-Harris KE, Durham C, Logan A, Smith G, DuBose-Morris $R$. Integration of telehealth education into the health care provider curriculum: a review. Telemed J E-Health. 2021;27(2):137-49. https://doi.org/10.1089/tmj.2019.0261.

59. Jumreornvong O, Yang E, Race J, Appel J. Telemedicine and medical education in the age of COVID-19. Acad Med. 2020;95(12):1838-43. https://doi.org/10.1097/ACM.0000000000 003711.

60. Walker C, Echternacht H, Brophy PD. Model for medical student introductory telemedicine education. Telemed J E-Health. 2019;25(8):717-23. https://doi.org/10.1089/tmj.2018.0140.

61. Roberts ET, Mehrotra A. Assessment of disparities in digital access among medicare beneficiaries and implications for telemedicine. JAMA Intern Med. 2020;180(10):1386-9. https://doi. org/10.1001/jamainternmed.2020.2666.

62. DeGuzman PB, Bernacchi V, Cupp CA, et al. Beyond broadband: digital inclusion as a driver of inequities in access to rural cancer care. J Cancer Surviv Res Pract. 2020;14(5):643-52. https://doi. org/10.1007/s11764-020-00874-y.

63. Muñoz-López C, Ramírez-Cornejo C, Marchetti MA, et al. Performance of a deep neural network in teledermatology: a single-centre prospective diagnostic study. J Eur Acad Dermatol Venereol. 2021;35(2):546-53. https://doi.org/10.1111/jdv.16979.

64. Blum A, Bosch S, Haenssle HA, et al. Künstliche intelligenz und smartphone-programm-applikationen (Apps). Hautarzt. 2020;71(9):691-8. https://doi.org/10.1007/s00105-020-04658-4.

65. Po (Harvey) Chin Y, Hsin Huang I, Yu Hou Z, et al. User satisfaction with a smartphone-compatible, artificial intelligence-based cutaneous pigmented lesion evaluator. Comput Methods Programs Biomed. 2020;195:105649. https://doi.org/10.1016/j.cmpb.2020. 105649 . 